\title{
Kinematic and Acoustic Similarities of Separated Turbulent Boundary Layers
}

\author{
Alexandre Suryadi* \\ German Aerospace Center (DLR), 38108 Braunschweig, Germany
}

\begin{abstract}
A similarity model for separated turbulent boundary layer is proposed as a function of the velocity associated with the maximum local mean shear $U_{m}=\sqrt{\tau_{m} / \rho}$. A linear relation between mean velocity and reduced wall-normal coordinate is observed for approximately 40-50\% of the boundary layer height. A corresponding similarity model of the far-field sound pressure levels was also derived. The proposed scaling law shows less variability than the classical trailing-edge noise scaling within a range of the freestream velocity and airfoil's angle of attack. In comparison to classical trailing-edge noise, the sound pressure level scaling of separated flow is tuned by a factor of $U_{m}^{2}$, a non-dimensional velocity variable associated with the maximum mean shear stress in the separated boundary layer.
\end{abstract}

\section{Nomenclature}

\begin{tabular}{|c|c|}
\hline$a_{\infty}$ & speed of sound \\
\hline$B$ & Schofield-Perry integral layer thickness \\
\hline$c$ & chord length \\
\hline$f$ & frequency \\
\hline$f_{m}$ & one-third octave band center frequency \\
\hline$L$ & position of $\tau_{m}$ from the profile's point of origin \\
\hline$L_{p, 1 / 3}$ & one-third octave band sound pressure level \\
\hline$l_{\text {ref }}$ & arbitrary reference length, $1 \mathrm{~m}$ \\
\hline$M$ & Mach number \\
\hline$P$ & mean pressure \\
\hline$q_{e}$ & local dynamic pressure, $q_{e}=0.5 \rho U_{e}^{2}$ \\
\hline$R e_{\delta}$ & Reynolds number, $R e_{\delta}=U_{e} \delta / \nu$ \\
\hline$S t$ & Strouhal number \\
\hline$U$ & mean streamwise velocity \\
\hline$U_{e}$ & mean streamwise velocity at boundary layer thickness \\
\hline$U_{m}$ & velocity associated with maximum shear stress, Eq. (1) \\
\hline$U_{\text {ref }}$ & arbitrary velocity scale, $1 \mathrm{~m} / \mathrm{s}$ \\
\hline$U_{s}$ & Schofield-Perry velocity scale \\
\hline$u_{\tau}$ & friction velocity, $u_{\tau}=\sqrt{\tau_{w} / \rho}$ \\
\hline$U_{Z S}$ & Zagarola-Smits velocity scale \\
\hline$u, v, w$ & fluctuating velocity components \\
\hline$y$ & wall-normal direction \\
\hline$\alpha$ & angle of attack \\
\hline$\beta_{Z S}$ & Zagarola-Smits Clauser parameter \\
\hline$\gamma_{Z S}$ & Zagarola-Smits Reynolds stress ratio \\
\hline$\gamma_{p}$ & fraction of time that the flow near the wall is in the downstream direction \\
\hline$\delta$ & boundary layer thickness \\
\hline$\delta_{0}$ & height of zero-velocity streamline \\
\hline
\end{tabular}

\footnotetext{
${ }^{*}$ Research Engineer, Institute of Aerodynamics and Flow Technology, Technical Acoustics. Lilienthalplatz 7, 38108 Braunschweig, Germany. alexandre.suryadi@dlr.de
} 


$\begin{array}{ll}\delta_{1} & \text { displacement thickness } \\ \eta & \text { reduced wall-normal coordinates, Eq. (7) } \\ \nu & \text { kinematic viscosity } \\ \hat{\Pi} & \text { wake parameter } \\ \rho & \text { density } \\ \tau_{m} & \text { maximum shear stress } \\ \tau_{w} & \text { wall shear stress } \\ \Phi_{p p} & \text { surface pressure spectrum } \\ \text { Subscripts } & \\ g & \text { geometric } \\ i & \text { inner region } \\ l & \text { linear region } \\ m & \text { maximum mean shear } \\ \text { max } & \text { maximum } \\ \text { model } & \text { model } \\ o & \text { outer region } \\ \infty & \text { freestream } \\ \text { Superscripts } & \\ * & \text { dimensional parameters } \\ + & \text { normalization with shear variables } \\ \text { Abbreviations } & \\ \text { M.A.E } & \text { mean absolute error }\end{array}$

\section{Introduction}

For fluid machinery to operate consistently, it is designed to avoid turbulent boundary layer separation. When it occurs flow separation might lead to stall and aerodynamic performance suffers. Therefore, it is essential to predict the onset of separation. The operational conditions of most fluid machinery are in the steady-state. However, wind turbine operations rely on the local and temporal atmospheric conditions. This transience could lead to the possibility of separated flow over the turbine blades. The transient conditions may be relatively short compared to the operational lifetime of a wind turbine, but flow separation can lead to increased noise that may further impact the surrounding neighborhoods. For providing noise safety margins in wind turbine certification, it is essential that the extraneous noise sources, which among them is due to flow separation, are included. The topic of this paper is to develop similarity scalings for later prediction of flow separation noise.

Classically, flow separation is defined from the point where the skin friction has diminished to zero. Simpson et al. ${ }^{1}$ describe flow separation as a process that develops gradually along the streamwise, instead of as an event. This process is expressed by the probability of the flow near the wall is in the downstream direction, $\gamma_{p}$.

- Incipient Detachment (ID): the reverse flow occurs $1 \%$ of the sampled time, $\gamma_{p}=0.99$

- Intermittent Transitory Detachment (ITD): the reverse flow occurs $20 \%$ of the sampled time, $\gamma_{p}=0.8$

- Transitory Detachment (TD): the reverse flow occurs $50 \%$ of the sampled time, $\gamma_{p}=0.5$

- Detachment (D): where the time-averaged wall shear stress is zero

The flow is said to be separated in the classical sense during and after transitory detachment. Clauser ${ }^{2}$ conducted the earliest work of similarity analysis of turbulent boundary layer under adverse pressure gradient, a contributing factor leading to flow separation. The other factor is skin friction. Measurements were performed in conditions where the mean streamwise pressure gradient is inversely proportional to the wall shear stress, $\tau_{w}$. This condition is defined by the Clauser parameter $\delta^{\prime} / \tau_{w} \mathrm{~d} P / \mathrm{d} x=$ constant, where $\delta^{\prime}$ is a characteristic length scale to represent the boundary layer thickness. Clauser solved a modified Falkner-Skan equation to obtain the velocity profiles of the boundary layer at different magnitudes of the pressure gradient. The point of separation was defined where $\mathrm{d} U / \mathrm{d} y(y=0)=0$. Following Clauser, Stratford ${ }^{3}$ and Townsend ${ }^{4}$ 
scaled the near-wall velocity profile with a function that depends on the mean streamwise pressure gradient to find the critical values that lead to flow separation. Mellor and Gibson, ${ }^{5}$ Yaglom, ${ }^{6}$ and Castillo et al. ${ }^{7}$ used the same concept to find the similarity of the outer region of the boundary layer velocity profile.

A different scaling approach for the prediction of flow separation eventuality was proposed by Perry and Schofield ${ }^{8}$ using scales based on the local maximum shear stress, $\tau_{m}$,

$$
\begin{aligned}
U_{m} & =\sqrt{\frac{\tau_{m}}{\rho}} \\
U_{s} & =8 \frac{B}{L} U_{m} \\
B & =2.86 \delta_{1} \frac{U_{e}}{U_{s}}
\end{aligned}
$$

where $U_{m}$ is a velocity scale based on $\tau_{m}, U_{s}$ is the Schofield-Perry velocity scale, $B$ is the Schofield-Perry integral layer thickness, $L$ is the distance of $\tau_{m}$ from the origin of profile, and $\delta_{1}$ is the displacement thickness. Schofield ${ }^{9}$ applied this similarity law to the separating flow of Simpson et al. ${ }^{10}$ data. He used locations in the transitory detachment region, where these datapoints are located at 130in. $<x<144$ in..

Maciel et al. ${ }^{11}$ studied the adverse pressure gradient turbulent boundary layer similarity in the manner of the Zagarola-Smits similarity. ${ }^{12}$ The equilibrium boundary layer conditions that need to be fulfilled for the similarity are constant Reynolds stress ratio, $\gamma_{Z S}$, constant Clauser parameter, $\beta_{Z S}$, and constant boundary layer growth

$$
\begin{aligned}
\gamma_{Z S} & =\frac{U_{Z S}}{U_{e}} \frac{\delta_{1}}{\delta}=\text { Constant } \\
\beta_{Z S} & =\frac{-\delta}{U_{Z S}} \frac{\mathrm{d} U_{e}}{\mathrm{~d} x}=\text { Constant } \\
\frac{\mathrm{d} \delta}{\mathrm{d} x} & =\text { Constant }
\end{aligned}
$$

In addition to predicting the onset of flow separation, Sandborn and Liu ${ }^{13}$ modeled the velocity profile at the point of separation and a position downstream of reattachment. Several measurements to classify the kinematic profiles of separating turbulent boundary layer have been performed using the non-flow intrusive laser doppler anemometry ${ }^{1,10,14}$ and the flying hot-wire anemometry ${ }^{15,16}$ that measured profiles upstream and downstream of detachment. However, the kinematic model of a separated turbulent boundary layer is still elusive.

\section{I.A. Schofield-Perry velocity similarity}

A mean flow similarity was modeled using functions derived from the maximum shear stress velocity, see Eq. (1). The model accurately describes the two-dimensional turbulent boundary layer in transitory detachment provided that the point of origin is shifted to the zero velocity streamline. ${ }^{9}$ See also Perry and Schofield $^{8}$ for the derivation of the similarity model. The separated flow model of Schofield is

$$
\frac{U_{e}-U}{U_{s}}=1-0.4\left(\frac{y}{B}\right)^{1 / 2}-0.6\left(\frac{\pi y}{B}\right)
$$

and near the wall the velocity profile is in the form

$$
\frac{U}{U_{e}}=0.47\left(\frac{U_{s}}{U_{e}}\right)^{3 / 2}\left(\frac{y}{\delta_{1}}\right)^{1 / 2}+1-\frac{U_{s}}{U_{e}}
$$

\section{I.B. Schüle-Rossignol velocity similarity}

Schüle and Rossignol ${ }^{17}$ used the reduced wall-normal coordinate with the point-of-origin at the zero velocity streamline. Their similarity profile of a detached two dimensional turbulent boundary layer is a modification of the Coles' law of the wake.

$$
\frac{U(\eta)}{U_{e}}=\hat{\Pi} f(\eta)+g(\eta)+h(\eta, \hat{\Pi})
$$


where $\hat{\Pi}$ is a wake parameter inspired by Coles' law of the wake. The three functions are given as

$$
\begin{aligned}
f(\eta) & =\sin ^{2}\left(\frac{\pi}{2} \eta\right) \\
g(\eta) & =\frac{a_{1}}{\pi-4}\left[2+(\pi-4) \eta-(\pi-2) \eta^{2}-2 \cos \left(\frac{\pi}{2} \eta\right)\right] \\
h(\eta, \hat{\Pi}) & =\frac{1-\hat{\Pi}}{2}[\tanh (\alpha \eta-\beta)+1]
\end{aligned}
$$

With the last function, $h(\eta, \hat{\Pi})$, is a non-physical function to fit the model of the boundary conditions with $\alpha=12.5$ and $\beta=9.5$ given heuristically. The wall-normal coordinate has its point-of-origin at $U(y)=0$

$$
\eta=\frac{y-\delta_{0}}{\delta-\delta_{0}}
$$

and the parameter $a_{1}$ is the non-dimensional kinematic shear at the point-of-origin

$$
a_{1}=\left.\frac{1}{U_{e}} \frac{\mathrm{d} U}{\mathrm{~d} \eta}\right|_{\eta=0}
$$

This similarity poses an open problem as the application of the boundary conditions

$$
U(\eta=0)=0 ; \quad U(\eta=1)=U_{e}
$$

leads to

$$
\begin{aligned}
h(\eta=0, \hat{\Pi})=0 ; \quad h(\eta=1, \hat{\Pi})=1-\hat{\Pi} ; \\
h^{\prime}(\eta=0, \hat{\Pi})=0 ; \quad h^{\prime}(\eta=1, \hat{\Pi})=0
\end{aligned}
$$

giving the value $\hat{\Pi}$ indeterminable.

\section{I.C. Trailing-edge noise scaling}

Another scaling that is important in the study of aeroacoustics is the surface pressure scaling, from which the far-field noise scaling can be derived. Several well-regarded works on this topic are the general study of airfoil self-noise by Brooks et al., ${ }^{18}$ the surface pressure measurement of attached turbulent boundary layer for trailing edge noise prediction by Brooks and Hodgson ${ }^{19}$ and the surface pressure measurement in a separating turbulent boundary layer by Simpson et al. ${ }^{20}$ The trailing-edge noise and flow separation noise scales with $U_{e}^{5},{ }^{18,19}$ whereas in Simpson et al. flow separation noise is normalized with $\tau_{m}^{2}$. The fifth-power law scales the classical trailing-edge noise well, but it does not perfectly scale flow separation noise. This contradiction indicates that the separated boundary layer may be governed by different parameters.

In the present study, separated flow cases of Simpson et al. ${ }^{1}$ and Gleyzes and Capbern ${ }^{21}$ are selected because they were measured using laser Doppler velocimetry, an unobtrusive measurement technique. For Simpson et al. cases where $\gamma_{p}<0.5$ are chosen, i.e., 144 in. $\leq x \leq 170$ in. and in Gleyzes and Capbern velocity profiles of an undisclosed airfoil known as airfoil A at $\alpha_{g}=13^{\circ}$ are used. In the case of Gleyzes and Capbern's dataset, the Clauser parameter at the region of interest is approximately zero because of $\mathrm{d} P / \mathrm{d} x \approx 0$. Furthermore, the airfoil/curved surface does not ensure the development of an equilibrium boundary layer condition. The similarity model in this study uses the shear velocity as defined by maximum mean shear, like the Schofield-Perry similarity. However, it avoids using the Schofield-Perry velocity and length scales.

\section{Similarity of the mean velocity profile}

This study proposes a similarity model using the maximum mean shear velocity $U_{m}$ and the reduced wallnormal coordinate, where the origin point is at the position of the zero velocity in the separated boundary layer. The flow is assumed to be incompressible, statistically stationary, and effectively two-dimensional. This section will detail the derivation of the model. First, the flow parameters are non-dimensionalized using $U_{e}^{*}$ and $\delta^{*}$. Then $U_{m}^{*}$ is defined, and the boundary layer parameters are normalized with it. Finally, the dataset used for building the model is presented. In this and the following sections, the dimensional variables are denoted by an asterisk, * . 


\section{II.A. Non-dimensionalized variables}

The mean streamwise velocity, $U^{*}$, and position in the wall-normal direction, $y^{*}$, are non-dimensionalized with the local freestream velocity, $U_{e}^{*}$, and the local boundary layer height, $\delta^{*}$, respectively.

$$
U=\frac{U^{*}}{U_{e}^{*}} ; \quad y=\frac{y^{*}}{\delta^{*}}
$$

The maximum shear velocity $U_{m}^{*}$ is given as

$$
U_{m}^{*}=\sqrt{\frac{\tau_{m}^{*}}{\rho^{*}}}=\sqrt{\left.\nu^{*} \frac{\mathrm{d} U^{*}}{\mathrm{~d} y^{*}}\right|_{\max }}
$$

\section{II.B. Shear normalized variables}

Similar to the law-of-the-wall the velocity profile is normalized by $U_{m}^{*}$ and the wall-normal direction by $U_{m}^{*} / \nu^{*}$. We will loosely symbolize these normalized variables as $U^{+}$and $y^{+}$.

$$
\begin{gathered}
U^{+}=\frac{U^{*}}{U_{m}^{*}}=\frac{U^{*}}{\sqrt{\nu^{*} \mathrm{~d} U^{*} / \mathrm{d} y_{\max }^{*}}} \\
=\sqrt{R e_{\delta}} \frac{U}{\sqrt{d U / d y_{\max }}} \\
y^{+}=\frac{y^{*} U_{m}^{*}}{\nu^{*}}=y \frac{\delta^{*}}{\nu^{*}} \sqrt{\frac{\nu^{*} U_{e}^{*}}{\delta^{*}}} \sqrt{\left.\frac{d U}{d y}\right|_{\max }} \\
=y \sqrt{\operatorname{Re}_{\delta}} \sqrt{\left.\frac{d U}{d y}\right|_{\max }}
\end{gathered}
$$

Where $\operatorname{Re}_{\delta}=U_{e}^{*} \delta^{*} / \nu^{*}$. The non-dimensional form of the maximum shear velocity can be defined as

$$
U_{m}=\sqrt{\frac{d U}{d y}}=\sqrt{\operatorname{Rex} \delta} \frac{U_{m}^{*}}{U_{e}^{*}}
$$

substituting Eq. (15) to Eqs. (13) and (14) leads to $U^{+}=\sqrt{R e_{\delta}} U / U_{m}$ and $y^{+}=y \sqrt{\operatorname{Re}_{\delta}} U_{m}$, respectively.

\section{II.C. Dataset}

Datasets from Simpson et al. ${ }^{1}$ and Gleyzes and Capbern ${ }^{21}$ are used as the reference in this study. Both of them used laser Doppler velocimetry to measure the velocity and turbulent stress profiles. These velocity profiles are shown in figure 1 in non-dimensional form. Typical boundary layer properties of the chosen

Table 1. Boundary layer parameters of profiles of Simpson et al. ${ }^{1}$ and Gleyzes and Capbern ${ }^{21}$

\begin{tabular}{llrrrrrrr}
\hline & & $\delta^{*},[\mathrm{~m}]$ & $\delta_{0}^{*},[\mathrm{~m}]$ & $U_{e}^{*},[\mathrm{~m} / \mathrm{s}]$ & $\operatorname{Re}_{\delta}$ & $U_{m}$ & $\delta_{0}$ & $-\delta_{0} U_{m}$ \\
\hline Simpson et al. & $x=144$ in. & 0.170 & 0.029 & 13.87 & 151471 & 1.258 & 0.170 & -0.213 \\
& $x=158$ in. & 0.237 & 0.059 & 13.59 & 206864 & 1.307 & 0.248 & -0.324 \\
& $x=170$ in. & 0.318 & 0.099 & 13.32 & 271482 & 1.376 & 0.310 & -0.426 \\
Gleyzes and Capbern & $x / c=0.87$ & 0.036 & 0.004 & 50.00 & 115385 & 1.234 & 0.100 & -0.124 \\
& $x / c=0.90$ & 0.041 & 0.005 & 50.00 & 132372 & 1.243 & 0.127 & -0.157 \\
& $x / c=0.93$ & 0.043 & 0.008 & 50.00 & 137821 & 1.234 & 0.178 & -0.220 \\
& $x / c=0.96$ & 0.051 & 0.010 & 50.00 & 163462 & 1.287 & 0.190 & -0.245 \\
& $x / c=0.99$ & 0.055 & 0.012 & 50.00 & 176282 & 1.290 & 0.215 & -0.277 \\
\hline
\end{tabular}




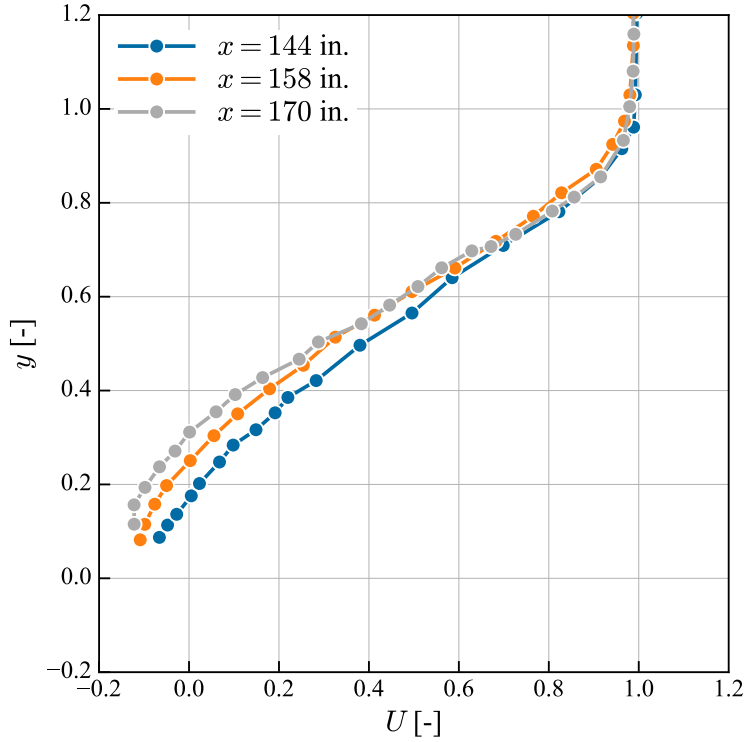

(a)

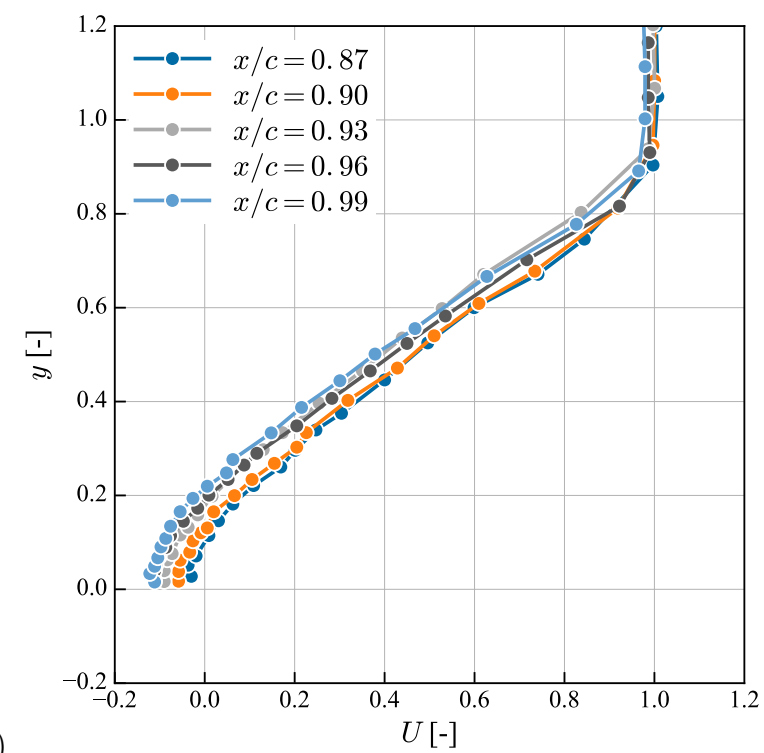

(b)

Figure 1. Velocity profiles from (a) Simpson et al. ${ }^{1}$ and (b) Gleyzes and Capbern ${ }^{21}$

dataset are presented in table 1 along with proposed variables $\delta_{0}^{*}$, the wall-normal position of the zero velocity and in its non-dimensional form, $\delta_{0}$, and $U_{m}$, the non-dimensional maximum shear velocity.

The values of $U_{m}$ were calculated from the dataset. A third-order spline function was applied to approximate a smooth mean velocity profile and the first derivative was then calculated from that function. The smoothing factor of the spline function is defined as

$$
\sum_{i}\left[\left(U_{i}-f\left(x_{i}\right)\right)\right]^{2} \leq 0.001
$$

with $U_{i}$ is the input data and $f\left(x_{i}\right)$ is the spline function.

The non-dimensional velocity profiles are shown in figure 2 with $U / U_{m}=U^{+} / \sqrt{\operatorname{Re}_{\delta}}$ as the abscissa and $\left(y-\delta_{0}\right) U_{m}=\left(y^{+}-\delta_{0}^{+}\right) \sqrt{\operatorname{Re}_{\delta}}$ as the ordinate. The relations described above produce a similarity profile from $\delta_{0}$ to $70-80 \%$ of $\delta$. Also, almost half of the velocity profile is linearly proportional to the reduced wall-normal coordinate. This linear region suggests that the separated turbulent boundary layer is mostly inviscid in the sense that the viscous force is approximately zero. Viscous forces become important around the edges of the boundary layer and closer to the surface. Based on these figures, a velocity profile model is proposed in the next section.

\section{Velocity profile model}

\section{III.A. Linear region}

The velocity profile of the linear region, $U_{l}$, can be expressed as

$$
\frac{\left(y^{+}-\delta_{0}^{+}\right)}{\sqrt{\operatorname{Re}_{\delta}}}=1.05 \frac{U_{l}^{+}}{\sqrt{\operatorname{Re}_{\delta}}}-0.070
$$

where $\delta_{0}^{+}=\sqrt{\operatorname{Re}_{\delta}} U_{m} \delta_{0}$. Equation (17) can be simplified using Eqs. (13)-(15).

$$
\left(y-\delta_{0}\right) U_{m}=1.05 \frac{U_{l}}{U_{m}}-0.070
$$

\section{III.B. Inner region}

The velocity profile just above $\delta_{0}$ is modeled as a velocity defect with respect to $U_{l}$. This profile is fitted with a sigmoid function in the form

$$
\frac{\Delta U_{i}}{U_{m}}=\frac{1}{a+b \exp (c \hat{\eta})}
$$




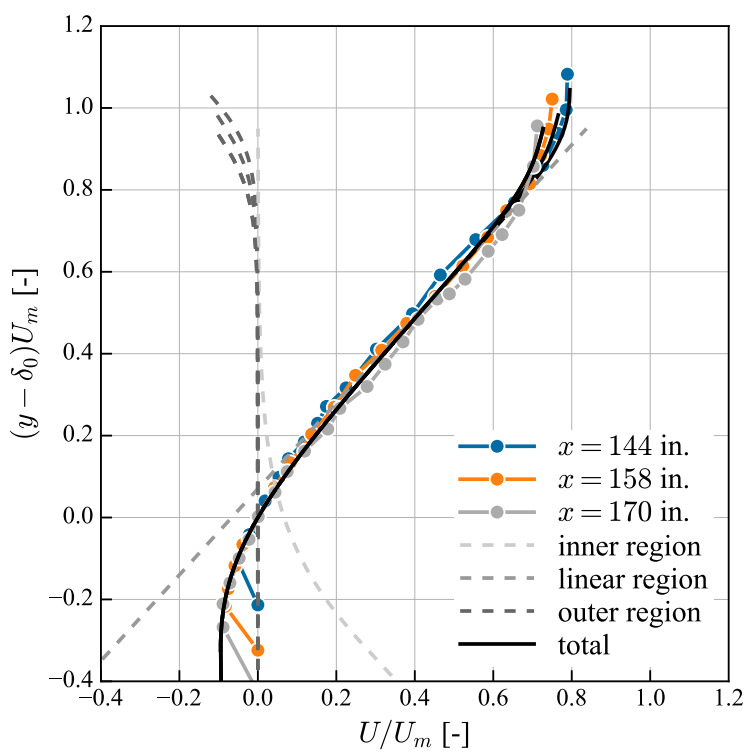

(a)

Figure 2. Proposed velocity similarity with profiles of (a) Simpson et al. ${ }^{1}$ and (b) Gleyzes and Capbern. ${ }^{21}$

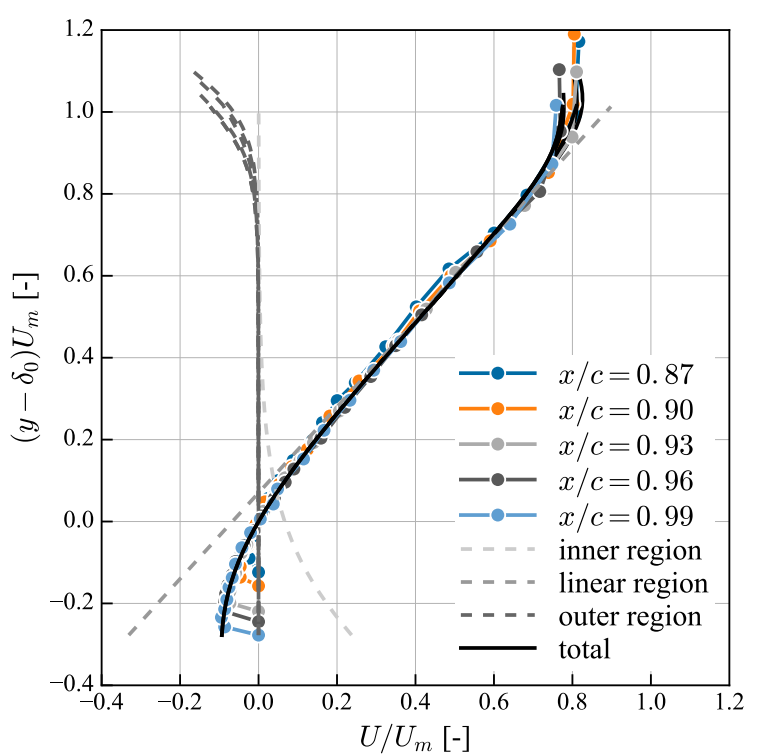

(b)$$
\text { . }
$$ 


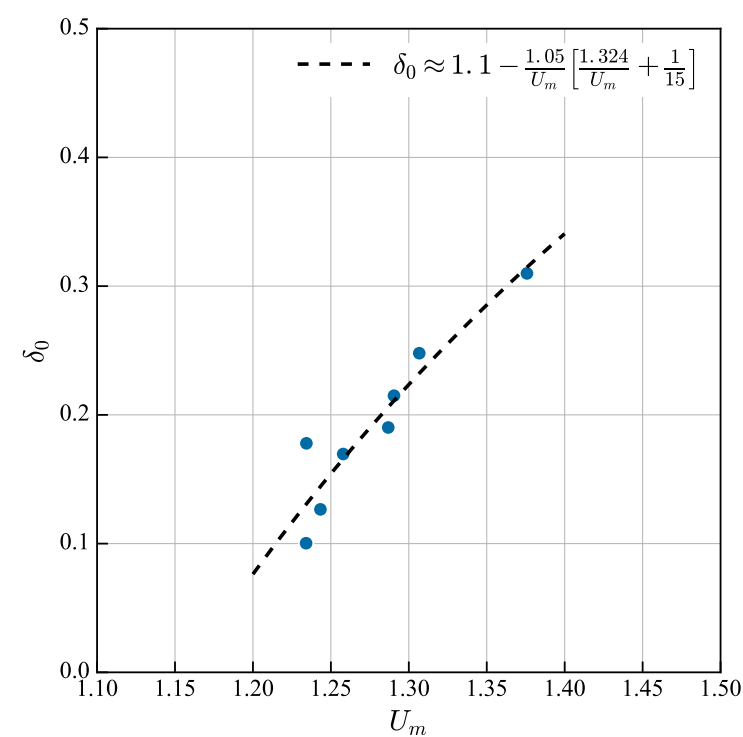

Figure 3. Relationship between $U_{m}$ and $\delta_{0}$

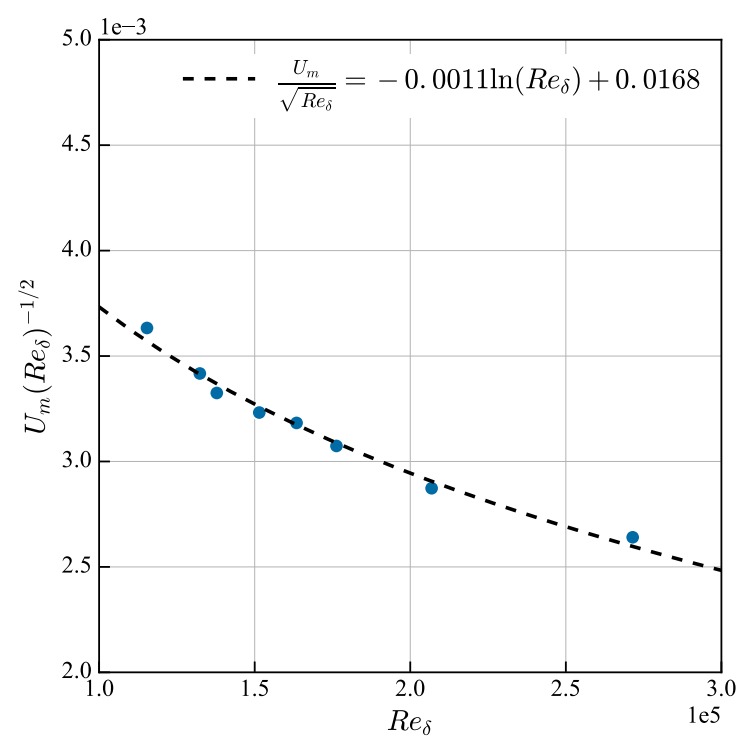

Figure 4. Relationship between $U_{m}$ and $R e_{\delta}$

\section{III.D. Relation between $U_{m}$ and $\delta_{0}$}

Based on the mathematical functions in the previous sections, a velocity profile can be reconstructed if the following parameters are known: $\delta_{0}, U_{m}$, and $R e_{\delta}$. In this and the next section, the relationship between these parameters are explored. By rearranging Eq. (18) and extrapolating it to $y=1.1$, the ensemble average of $U_{l}$ is $\left\langle U_{l}\right\rangle=1.324$ with standard of deviation $\sigma_{U_{l}}=0.033$. The value $y=1.1$ was selected because it provides the minimum least-square error from the available dataset.

$$
\begin{aligned}
\left(y-\delta_{0}\right) U_{m} & =1.05 \frac{U_{l}}{U_{m}}-0.07 \\
\delta_{0} & =y-\frac{1.05}{U_{m}}\left[\frac{U_{l}}{U_{m}}+\frac{1}{15}\right] \\
\delta_{0} & \approx 1.1-\frac{1.05}{U_{m}}\left[\frac{1.324}{U_{m}}+\frac{1}{15}\right]
\end{aligned}
$$

Furthermore, by using the boundary condition on the wall $y=0 \rightarrow y^{+}=0$ the left hand side of Eq. (18)

$$
-\delta_{0} U_{m}<0
$$

because $\delta_{0}>0$ and $U_{m}>0$. Substituting Eq. (26) to Eq. (25) gives

$$
\begin{array}{r}
-\left[1.1-\frac{1.05}{U_{m}}\left(\frac{1.324}{U_{m}}-\frac{1}{15}\right)\right] U_{m}<0 \\
1.1 U_{m}^{2}+0.07 U_{m}-1.39>0
\end{array}
$$

The first solution $U_{m}$ is negative, so that a requirement of the model is $U_{m}>1.156$. It is important to note that this value is only an approximate value as the minimum least-square method was used to close Eq. (18).

\section{III.E. Relation between $U_{m}$ and $R e_{\delta}$}

From the dataset, $U_{m}$ and $R e_{\delta}$ can be fitted with a logarithmic line as shown in figure 4 .

$$
\frac{U_{m}}{\sqrt{R e_{\delta}}}=\frac{U_{m}^{*}}{U_{e}^{*}}=-0.0011 \ln \left(R e_{\delta}\right)+0.0168
$$

Hence, from both relations of $U_{m}\left(\delta_{0}\right)$ in Eq. (25) and $U_{m}\left(R e_{\delta}\right)$ in Eq. (28), a velocity profile can be modeled based on the knowledge of the value of $R e_{\delta}$. 


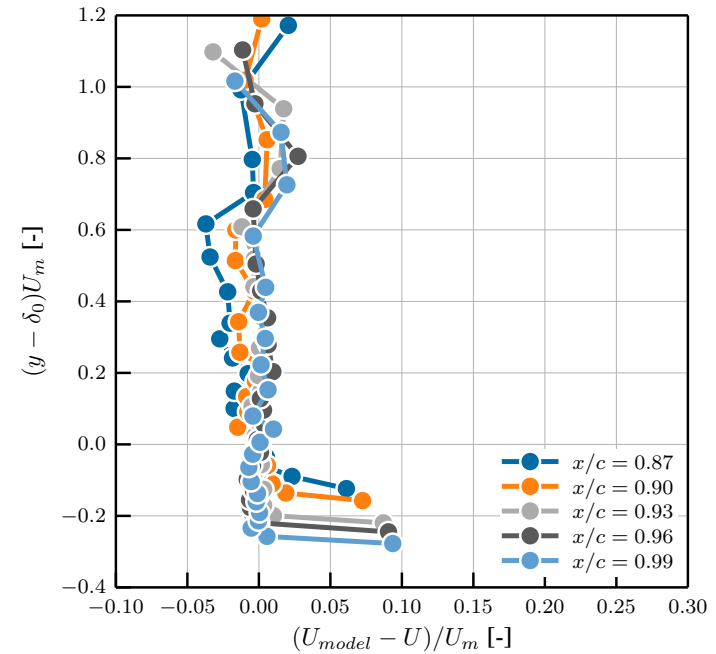

(a)

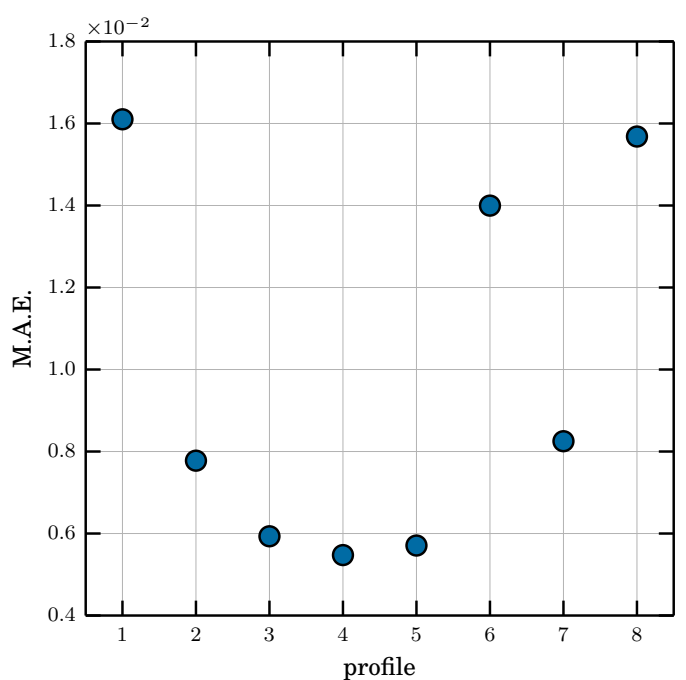

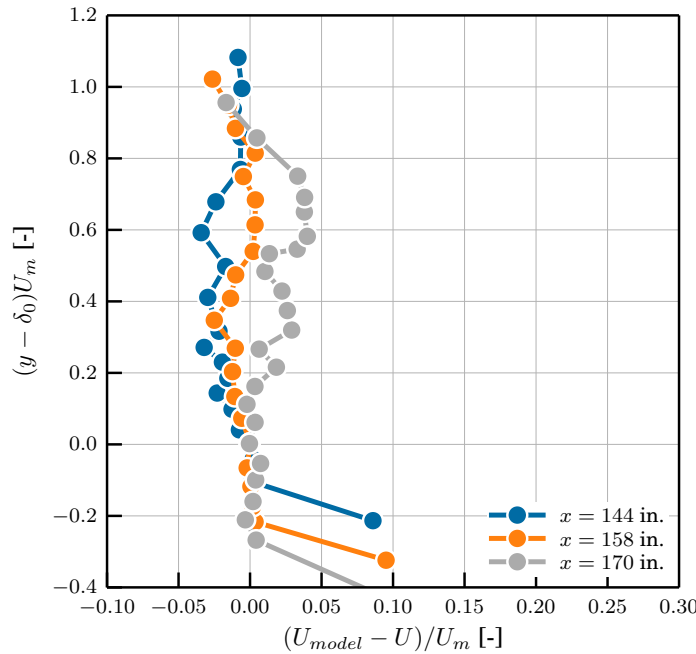

(b)

(c)

Figure 5. Comparison of modeled and measured velocity profile of (a) Gleyzes and Capbern and (b) Simpson et al. (c) mean absolute error of the velocity profile model. Profiles 1-5: Gleyzes and Capbern, profiles 6-8: Simpson et al.

\section{III.F. Error analysis of the proposed model}

The model velocity profiles, $U_{\text {model }}$, calculated based on the value of $R e_{\delta}$ from table 1 are compared with the measured velocity profiles, $U$. For $\left(y-\delta_{0}\right) U_{m}<0$ in figure $5(\mathrm{a})-(\mathrm{b})$ the velocity difference is larger than the rest of the profile because the model does not predict well the velocity next to the wall. The valid prediction range is considered for $\left(y-\delta_{0}\right) U_{m} \geq 0$.

Within the valid prediction range, the sectional error of the velocity profile is at most $5 \%$ of the measured velocity profile. The mean absolute error of the model is described in Eq. (29) and is shown to be less than $2 \%$ of the local freestream velocity in figure $5(\mathrm{c})$.

$$
\text { M.A.E }=\frac{1}{N} \sum_{i=1}^{N}\left|U_{\text {model }}\left(y_{i}\right)-U\left(y_{i}\right)\right|
$$

\section{Turbulent stress similarity and model}

Turbulent stress profiles $\langle u u\rangle,\langle v v\rangle$, and $-\langle u v\rangle$ are normalized in the same steps as the turbulent velocity profiles 


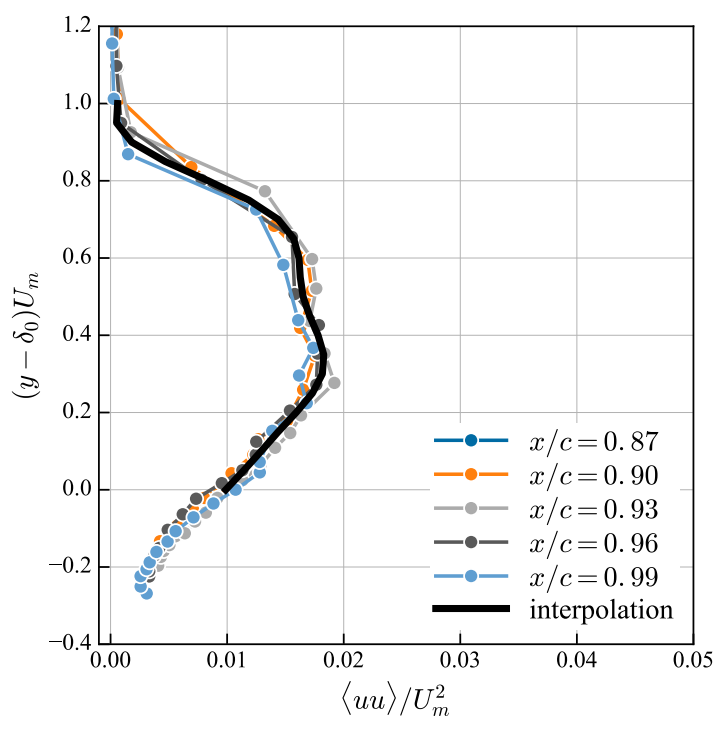

(a)

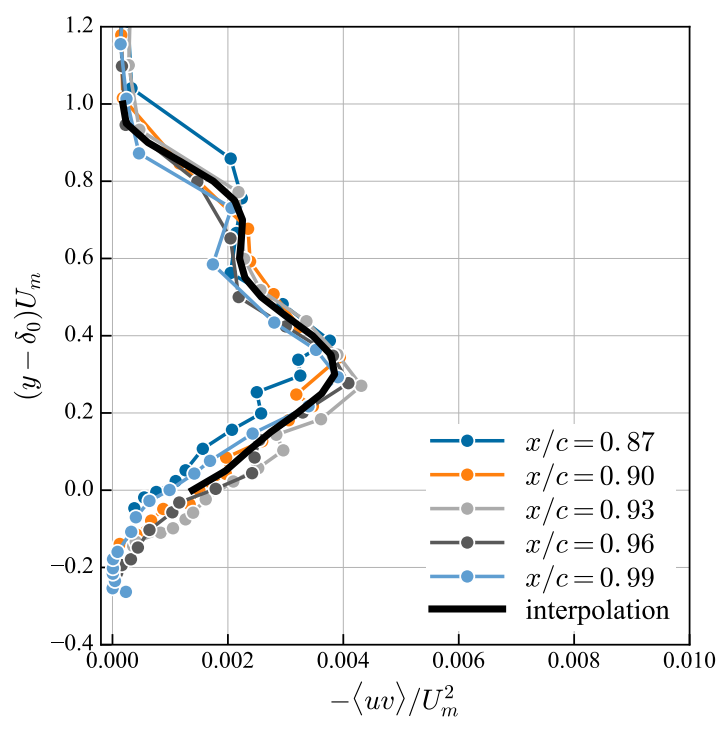

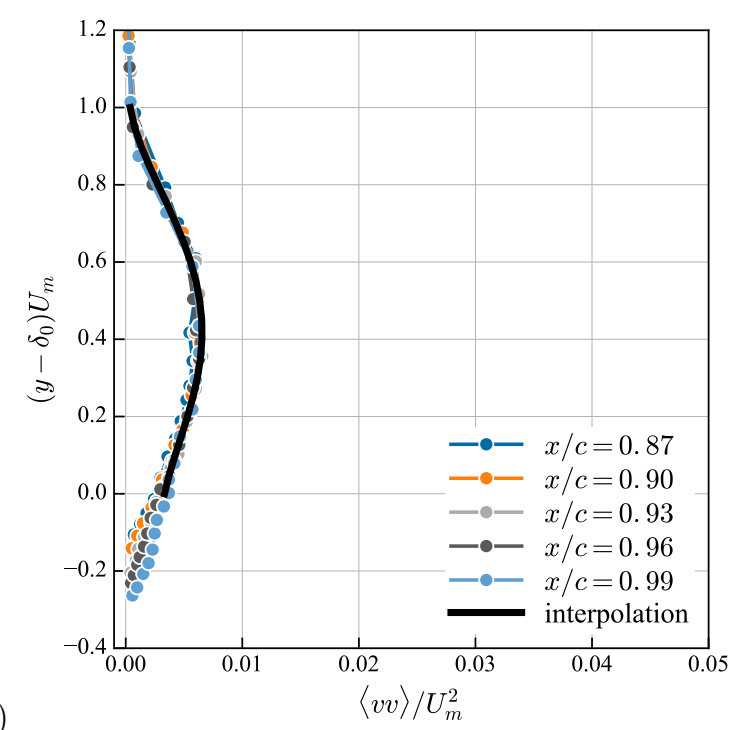

(b)

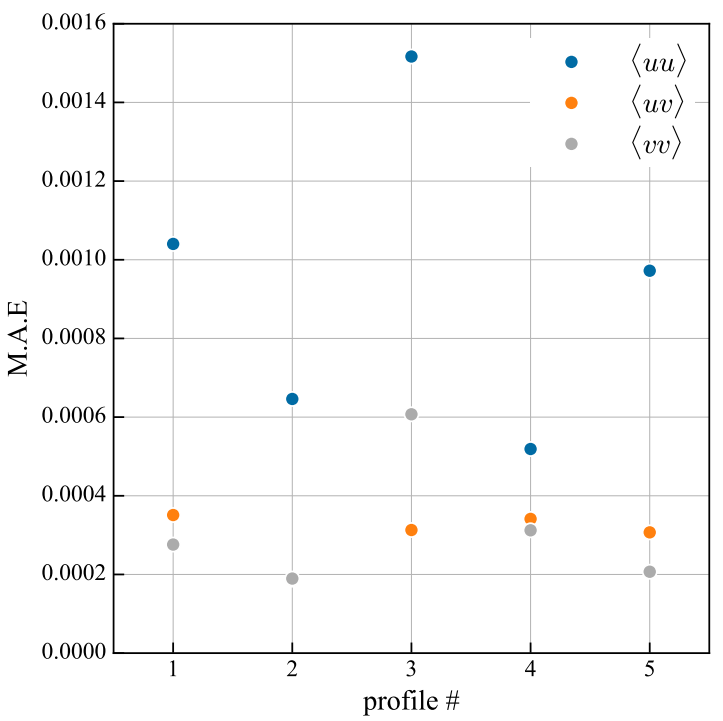

(d) (a) $\langle u u\rangle$, (b) $\langle v v\rangle$, (c) $-\langle u v\rangle$, and (d) mean absolute error

Figure 6. Turbulent stress profiles of Gleyzes and Capbern: ${ }^{21}$ of the turbulent stress models.

$$
\begin{gathered}
\left\langle u^{+} u^{+}\right\rangle=\frac{\left\langle u^{*} u^{*}\right\rangle}{U_{m}^{* 2}}=\frac{\left\langle u^{*} u^{*}\right\rangle}{U_{e}^{* 2} U_{m}^{2}} R e_{\delta}=\frac{\langle u u\rangle}{U_{m}^{2}} R e_{\delta} \\
\left\langle v^{+} v^{+}\right\rangle=\frac{\left\langle u^{*} u^{*}\right\rangle}{U_{m}^{* 2}}=\frac{\left\langle v^{*} v^{*}\right\rangle}{U_{e}^{* 2} U_{m}^{2}} R e_{\delta}=\frac{\langle v v\rangle}{U_{m}^{2}} R e_{\delta} \\
-\left\langle u^{+} v^{+}\right\rangle=-\frac{\left\langle u^{*} u^{*}\right\rangle}{U_{m}^{* 2}}=-\frac{\left\langle u^{*} v^{*}\right\rangle}{U_{e}^{* 2} U_{m}^{2}} R e_{\delta}=-\frac{\langle u v\rangle}{U_{m}^{2}} R e_{\delta}
\end{gathered}
$$

The profiles are plotted with the reduced wall-normal coordinate $\left(y-\delta_{0}\right) U_{m}$. The profiles $\langle u u\rangle / U_{m}^{2}$ and $-\langle u v\rangle / U_{m}^{2}$ can be fitted with a $10^{\text {th }}$ order polynomial and the profile for $\langle v v\rangle / U_{m}^{2}$ with a $4^{\text {th }}$ order polynomial. The increase of the order of the polynomial is mainly due to the inflection point at $\left(y-\delta_{0}\right) U_{m} \approx 0.6$. The mean absolute error for each profile is shown in figure $6(\mathrm{~d})$ and the overall mean absolute errors are $9.4 \times 10^{-4}$ for $\langle u u\rangle, 3.2 \times 10^{-4}$ for $\langle v v\rangle$, and $3.0 \times 10^{-4}$ for $-\langle u v\rangle$. 


\section{Similarity of the far-field acoustic pressure}

The far-field sound of separated turbulent boundary layers of the DU96-W-180 airfoil was measured at three geometric angles of attack, $\alpha_{g}=11^{\circ}, 13^{\circ}, 14.7^{\circ}$ and three freestream velocities $U_{\infty}^{*}=40,50$, and $60 \mathrm{~m} / \mathrm{s}$. For a description of the measurement setup, see Schüle and Rossignol. ${ }^{22}$ The far-field acoustic was measured using a directional microphone that provides trustworthy sound pressure levels for $1<f_{m}^{*}<10 \mathrm{kHz}$. Hotwire anemometry was used to determine the boundary layer thicknesses at $U_{\infty}^{*}=60 \mathrm{~m} / \mathrm{s}$. Because of the directional bias inherent in the hot-wire anemometry system, upstream moving flow cannot be distinguished from the downstream moving one. The determination of $U_{m}$ was then performed by calculating the flow shear at $0.4<y^{*} / \delta^{*}<1$ and compared with Eq. (28). There is a maximum of $10 \%$ difference between the measured and modeled $U_{m}$. This leads to the values $U_{m}$ presented in table 2 along with $U_{e}^{*}$, $\delta^{*}$, and $R e_{\delta}$

There is a variety of scaling laws for sound pressure levels, $L_{p, 1 / 3} \cdot{ }^{24}$ The experimentalists can scale their data based on $U_{e}^{*}, \delta_{1}^{*}$ or $u_{\tau}^{*}, \delta^{*}$, where $\delta_{1}^{*}$ is the displacement thickness. A third scaling law is proposed here based on $U_{e}^{*}, \delta^{*}$, and $U_{m}$. With $\left\langle p^{* 2}\right\rangle=M^{* 2} \Phi_{p p}(f)$, where $\left\langle p^{* 2}\right\rangle$ is the far-field sound pressure, $\Phi_{p p}$ is the power spectral density of the surface pressure and $M^{*}=U_{e}^{*} / a_{\infty}^{*}$ is the Mach number, the scaling of the sound pressure levels can be derived according to the scaling of $\Phi_{p p}(f)$.

\section{V.A. Scaling with freestream properties}

Canonically the surface pressure spectrum scales with $\Phi_{p p}(f) \propto q_{e}^{* 2} \delta^{*} / U_{e}^{*}$, where $q_{e}^{*}=0.5 \rho^{*} U_{e}^{* 2}$. The normalized sound pressure level is

$$
\begin{aligned}
L_{p, 1 / 3 ; 1} & =L_{p, 1 / 3}-50 \log M-10 \log \delta^{*} / l_{\text {ref }} \\
S t_{1} & =f^{*} \delta^{*} / U_{e}^{*}
\end{aligned}
$$

where $l_{\text {ref }}=1 \mathrm{~m}$ is an arbitrary reference length to normalize the boundary layer thickness.

\section{V.B. Scaling with mean shear properties}

In attached turbulent boundary layer the surface pressure spectrum scales with $\Phi_{p p}(f) \propto \tau_{w}^{* 2} \delta_{1}^{*} / U_{e}^{*}$. When the boundary layer separates, $\tau_{w}^{* 2}$ is represented by $\tau_{m}^{* 2}$ and $\delta_{1}^{*}$ by $\delta^{*}$, then $\Phi_{p p}(f)$ scales as $\Phi_{p p}(f) \propto$ $\tau_{m}^{* 2} \delta^{*} / U_{e}^{*}$. The second normalization of the sound pressure level is

$$
\begin{aligned}
L_{p, 1 / 3 ; 2} & =L_{p, 1 / 3}-10 \log (M)-40 \log \left(U_{m}^{*} / U_{r e f}\right)-10 \log \delta^{*} / l_{\text {ref }} \\
S t_{2} & =f^{*} \delta^{*} / U_{e}^{*}=S t_{1}
\end{aligned}
$$

where $U_{r e f}=1 \mathrm{~m} / \mathrm{s}$ is an arbitrary velocity scale of unit value to normalize $U_{m}^{*}$.

\section{V.C. Scaling with mixed properties}

With the scaling introduced for the mean velocity profile in Eqs. (13) and (14), the Strouhal number is rewritten as

$$
\begin{aligned}
f^{*} & \propto \frac{U_{e}^{*}}{\delta^{*}}=\frac{U_{e}^{+} U_{e}^{*} U_{m}}{\sqrt{R e_{\delta}}} \cdot \frac{U_{m} \sqrt{R e_{\delta}}}{\delta^{+} \delta^{*}} \\
f^{*} & \propto \frac{U_{e}^{+}}{\delta^{+}} \frac{U_{e}^{*}}{\delta^{*}} U_{m}^{2} \\
\frac{f^{*} \delta^{*}}{U_{e}^{*}} & \propto \frac{U_{e}^{+}}{\delta^{+}} U_{m}^{2} \\
S t & \propto f^{+} U_{m}^{2}
\end{aligned}
$$

Table 2. Boundary layer parameters of profiles of Suryadi and Herr. ${ }^{23}$

\begin{tabular}{llrrrrr}
\hline & & $\delta^{*},[\mathrm{~m}]$ & $U_{e}^{*},[\mathrm{~m} / \mathrm{s}]$ & $\operatorname{Re}_{\delta}$ & $U_{m}$ & $U_{m}$, Eq. $(28)$ \\
\hline Suryadi and Herr & $\alpha_{g}=11^{\circ}$ & 0.020 & 56.9 & 71125 & 1.164 & 1.20 \\
& $\alpha_{g}=13^{\circ}$ & 0.029 & 59.0 & 109679 & 1.324 & 1.27 \\
& $\alpha_{g}=14.7^{\circ}$ & 0.039 & 61.5 & 153750 & 1.339 & 1.30 \\
\hline
\end{tabular}


where $f^{+}$is a non-dimensional frequency and $U_{m}^{2}$ is a non-dimensional time scale as a result of the proposed mixed scaling.

The mixed scale power spectra can be derived from Parseval's theorem

$$
\begin{gathered}
\overline{p^{* 2}}=\int_{-\infty}^{\infty} \Phi_{p p}^{*}\left(f^{*}\right) \mathrm{d} f^{*} \\
\overline{p^{2}}=\frac{\overline{p^{* 2}} U_{e}^{*}}{0.5 \rho^{* 2} U_{e}^{* 4} \delta^{*}}=\int_{-\infty}^{\infty} \Phi_{p p}(S t) \mathrm{d} S t \\
\overline{p^{2}}=\frac{\overline{p^{* 2}} U_{e}^{*}}{0.5 \rho^{* 2} U_{e}^{* 4} \delta^{*}}=\int_{-\infty}^{\infty} \Phi_{p p}(S t) \mathrm{d} f^{+} U_{m}^{2} \\
\overline{p^{2}}=\frac{\overline{p^{* 2}} U_{e}^{*}}{0.5 \rho^{* 2} U_{e}^{* 4} \delta^{*}}=\int_{-\infty}^{\infty} U_{m}^{2} \Phi_{p p}(S t) \mathrm{d} f^{+}
\end{gathered}
$$

where $\overline{p^{* 2}}$ is the fluctuating surface pressure. Define $\Phi_{p p}^{+}\left(f^{+}\right)=U_{m}^{2} \Phi_{p p}(S t) \propto U_{e}^{*} U_{m}^{2} / \rho^{* 2} U_{e}^{* 4} \delta^{*} \Phi_{p p}^{*}(f)$ and substituting to Eq. (31) leads to the normalization of the sound pressure level with mixed scales

$$
\begin{aligned}
L_{p, 1 / 3 ; 3} & =L_{p, 1 / 3}-50 \log (M)+20 \log \left(U_{m}\right)-10 \log \left(\delta^{*} / l_{\text {ref }}\right) \\
f^{+} & =S t_{1} / U_{m}^{2}
\end{aligned}
$$

The normalizations according to Eqs. (31), (32), and (35) are plotted in figure 7. It shows that the proposed similarity model scales the far-field noise spectra better than the other two scaling models. The unscaled far-field noise spectra for constant $U_{\infty}$ shows a broadband increase from $\alpha_{g}=13^{\circ}$ to $\alpha_{g}=14.7^{\circ}$. After normalization with Eq. (35), this is shown to be dependent on the outer scale parameters $U_{e}^{*}, \delta^{*}$ and $U_{m}$. A kink at $f^{+}=1$ is observed in the far-field noise spectrum. This kink is also observable in the experimental data by Brooks et al. ${ }^{18}$ Unfortunately, the very interesting spectral peak amplitude and frequency are outside the measurable range of the measurement system, because that peak frequency is relatively lower than that of the canonical turbulent boundary layer flows. This lower limit is the challenge in the prediction of noise for separated turbulent boundary layer.

Measurement of surface pressure fluctuations of the DU96-W-180 had been conducted in the same facility as the measurement of far-field noise. ${ }^{23}$ Figure 8 is the surface pressure autospectra for separated angles of attack scaled using (a) freestream properties and (b) using Eqs. (33) and (33) and calculated for the 1/3 octave frequency bands.

$$
\overline{p^{* 2}}\left(f_{m}^{*}\right)=\int_{f_{l}^{*}}^{f_{u}^{*}} \Phi_{p p}^{*}\left(f^{*}\right) \mathrm{d} f^{*}
$$

where $f_{u}^{*}=2^{1 / 2} f_{m}^{*}, f_{l}^{*}=2^{-1 / 2} f_{m}^{*}$ and $f_{m}^{*}$ is the center frequency. The difference between the two scaling methods is almost negligible. However, spectral similarities between figure 7 (d) and $8(\mathrm{~b})$ for $f^{+}<1$ is quite uncanny. Notably, the kink in the far-field noise is observed at $f^{+} \approx 0.7$ at the same $f^{+}$where the surface pressure spectra also have a kink. Furthermore, figure 8(b) captures the peak of the spectra, which is an encouraging development for the prediction of the flow separation noise on the basis of the surface pressure prediction. For $f^{+}>1$ in figure 8(b), high frequency increase of the spectral level is observed at $\alpha_{g} \geq 14^{\circ}$ that is a divergence from the far-field noise spectra, which will be a topic for another publication.

\section{Summary and outlook}

A velocity scaling law is proposed with the velocity associated with the local maximum mean shear stress. In this case, only the streamwise time-averaged velocity component is considered. The self-similarity parameters are derived from this basis.

The proposed velocity scaling law agrees well with two datasets: the turbulent boundary layer at streamwise positions over an airfoil and in a diverging channel. A linear velocity relation encompassing a region with approximately $40-50 \%$ of the boundary layer height suggests that the separated turbulent boundary layer is mostly inviscid in the sense that the viscous force is approximately zero. Dissipation becomes important around the edges of the boundary layer and closer to the surface. With the same self-similarity parameters, the scaling of the separated turbulent shear stress profiles on the surface of an airfoil agrees well. Polynomial relations were derived to model $\langle u u\rangle,\langle v v\rangle,-\langle u v\rangle$. 


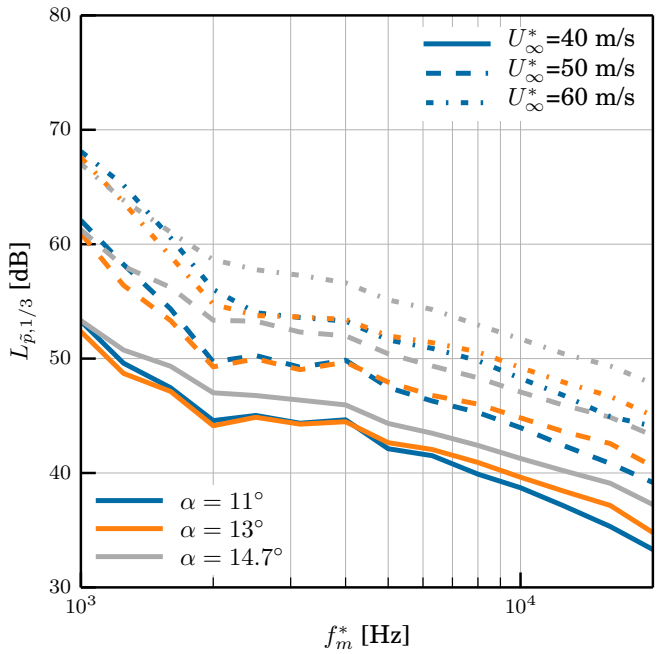

(a)

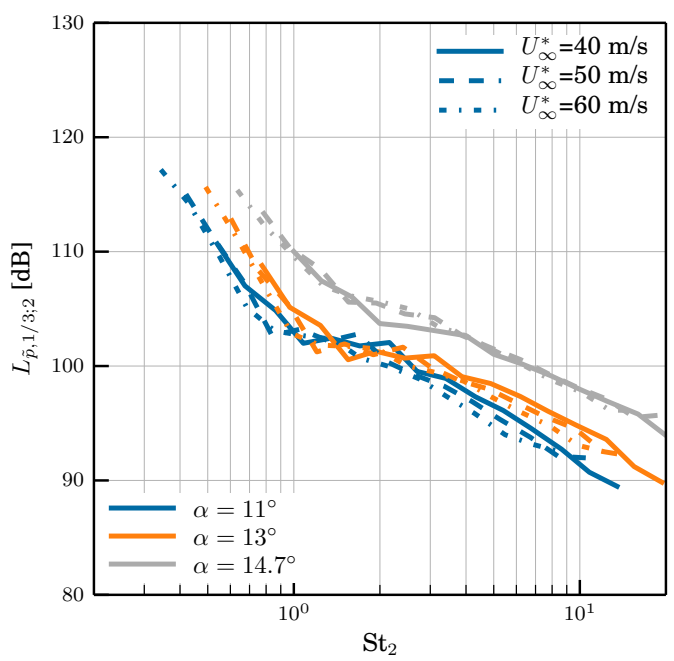

(c)

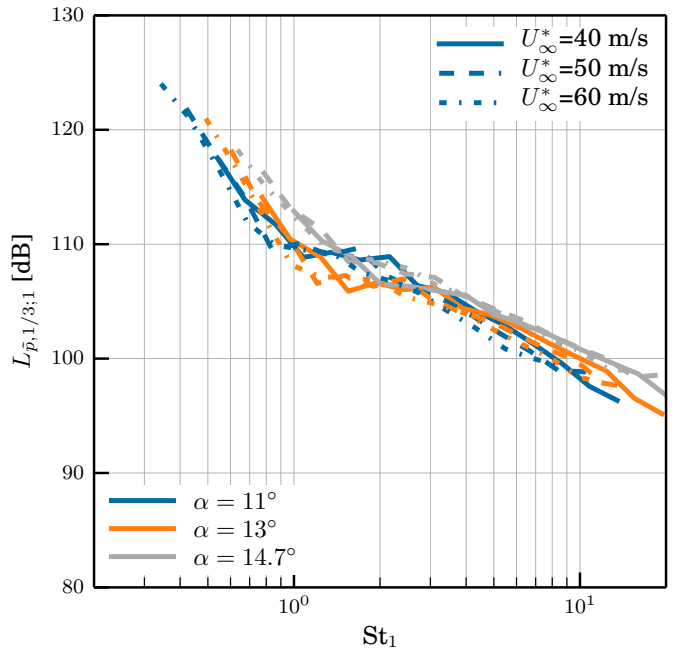

(b)

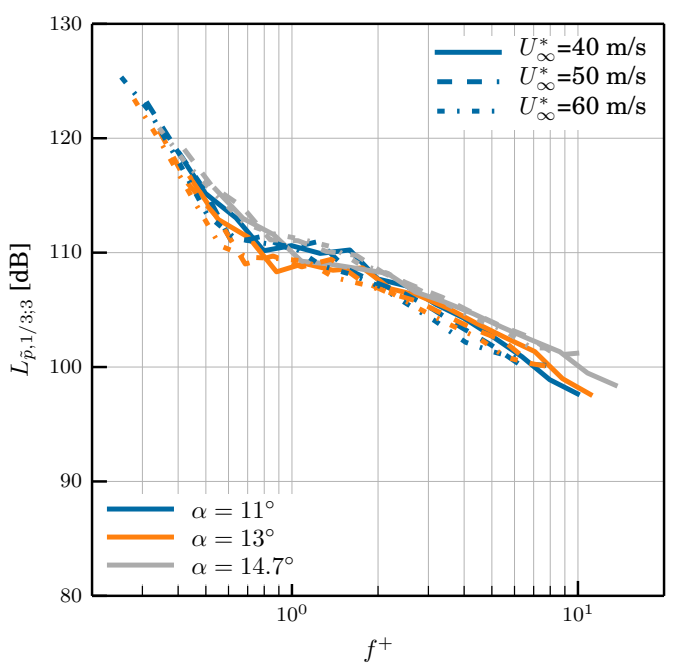

Figure 7. Unscaled and scaled sound pressure levels (a) Unscaled, (b) Eq. (31), (c) Eq. (32), and (d) Eq. (35)

The self-similarity of the far-field sound pressure levels is derived with the same methodology. Despite this similarity derivation is based on the streamwise component, there is a good agreement between the sound pressure level spectra of different freestream velocities and angles of attack. Compared to the classical trailing-edge noise, the proposed scaling of the far-field noise is tuned by a factor of $U_{m}^{2}$, a value derived from the mean velocity profile.

The end goal of this study is to formulate a prediction model for the far-field noise of a separated turbulent boundary layer. This prediction is necessary in cases with flow transience, for example, the flow separation over wind turbine blades due to a wind gust or other contributing atmospheric conditions. Suryadi and Herr ${ }^{23}$ made measurements of surface pressure for separated turbulent boundary layer. Those measurements also scale well with the proposed scaling in the frequency range of interest. On this basis, a far-field noise prediction scheme can be derived from a surface pressure prediction scheme like the TNO model by way of scaling the kinematic properties of the TNO model according to the similarities proposed in this study. That prediction scheme will be left for discussion in upcoming papers. 


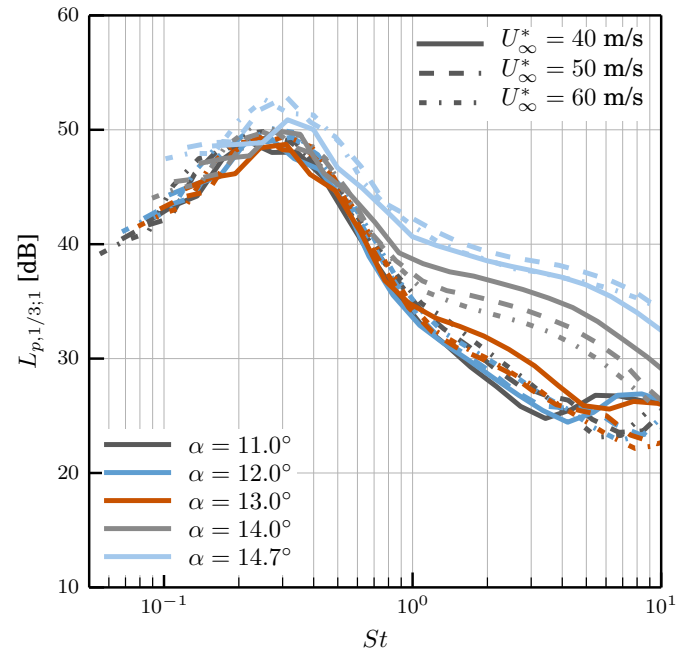

(a)

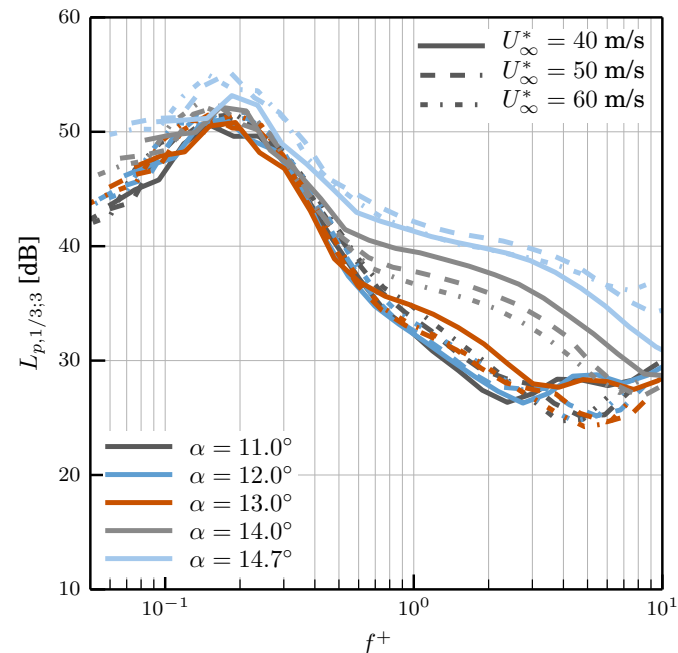

(b)

Figure 8. Surface pressure autospectra scaled using (a) freestream properties and (b) Eqs. (33) and (33).

\section{References}

\footnotetext{
${ }^{1}$ Simpson, R. L., Chew, Y.-T., and Shivaprasad, B. G., "The structure of a separating turbulent boundary layer .Part 1 . Mean flow and Reynolds stresses," Journal of Fluid Mechanics, Vol. 113, 1981, pp. 23-51.

${ }^{2}$ Clauser, F. H., "Turbulent Boundary Layers in Adverse Pressure Gradients," Journal of Aeronautical Sciences, Vol. 21, 1954, pp. 91-108.

${ }^{3}$ Stratford, B. S., "The prediction of separation of the turbulent boundary layer," Journal of Fluid Mechanics, Vol. 5, No. 1, 1959, pp. 1-16.

${ }^{4}$ Townsend, A. A., "The behaviour of a turbulent boundary layer near separation," Journal of Fluid Mechanics, Vol. 12, No. 4, 1962, pp. 536-554.

${ }^{5}$ Mellor, G. L. and Gibson, D. M., "Equilibrium turbulent boundary layers," Journal of Fluid Mechanics, Vol. 24, No. 2, 1966, pp. 225-253.

${ }^{6}$ Yaglom, A. M., "Similarity laws for constant-pressure and pressure-gradient turbulent wall flows," Annual Review of Fluid Mechanics, Vol. 11, 1979, pp. 505-540.

${ }^{7}$ Castillo, L. and George, W. K., "Separation Criterion for Turbulent Boundary Layers Via Similarity," Journal of Fluids Engineering, Vol. 126, 2004, pp. 297-304.

${ }^{8}$ Perry, A. E. and Schofield, W. H., "Mean velocity and shear stress distributions in turbulent boundary layers," Physics of Fluids, Vol. 16, No. 12, 1973, pp. 2068-2074.

${ }^{9}$ Schofield, W. H., "Two-dimensional separating turbulent boundary layers," AIAA Journal, Vol. 24, No. 10, 1986, pp. 1611-1620.

${ }^{10}$ Simpson, R. L., Chew, Y.-T., and Shivaprasad, B. G., "The structure of a separating turbulent boundary layer. Part 2. Higher-order turbulence results," Journal of Fluid Mechanics, Vol. 113, 1981, pp. 53-73.

${ }^{11}$ Maciel, Y., Rossignol, K.-S., and Lemay, J., "A Study of a Separated Turbulent Boundary Layer in Stalled-Airfoil-Type Flow Conditions," $35^{t h}$ AIAA Fluid Dynamics Conference and Exhibit, Toronto, Ontario. Canada, AIAA 2005-5291, 2005.

${ }^{12}$ Zagarola, M. V. and Smits, A. J., "Mean-flow scaling of turbulent pipe flow," Journal of Fluid Mechanics, Vol. 373, 1998, pp. 33-79.

${ }^{13}$ Sandborn, V. A. and Liu, C. Y., "On turbulent boundary-layer separation," Journal of Fluid Mechanics, Vol. 32, No. 02, 1968, pp. 293.

${ }^{14}$ Simpson, R. L., Strickland, J. H., and Barr, P. W., "Features of a separating turbulent boundary layer in the vicinity of separation," Journal of Fluid Mechanics, Vol. 79, No. 3, 1977, pp. 553-594.

${ }^{15}$ Coles, D. and Wadcock, A. J., "Flying-Hot-Wire Study of Flow Past an NACA 4412 Airfoil at Maximum Lift," AIAA journal, Vol. 17, No. 4, 1979, pp. 321-329.

${ }^{16}$ Thompson, B. E. and Whitelaw, J. H., "Characteristics of a trailing-edge flow with turbulent boundary-layer separation," Journal of Fluid Mechanics, Vol. 157, 1985, pp. 305-326.

${ }^{17}$ Schüle, C. Y. and Rossignol, K.-S., "A Separated Flow Model for Semi-Empirical Prediction of Trailing Edge Noise," Vol. 124 of Notes on Numerical Fluid Mechanics and Multidisciplinary Design, Springer International Publishing, 2014, pp. 639-647.

${ }^{18}$ Brooks, T. F., Pope, S., and Marcolini, M. A., "Airfoil Self-Noise and Prediction," NASA-RP-1218, NASA Langley Research Center; Hampton, VA, United States, 1989.

${ }^{19}$ Brooks, T. F. and Hodgson, T., "Trailing edge noise prediction from measured surface pressures," Journal of sound and vibration, Vol. 78, No. 1, 1981, pp. 69-117.
} 
${ }^{20}$ Simpson, R. L., Ghodbane, M., and McGrath, B. E., "Surface pressure fluctuations in a separating turbulent boundary layer," Journal of Fluid Mechanics, Vol. 177, 1987, pp. 167-186.

${ }^{21}$ Gleyzes, C. and Capbern, P., "Experimental study of two AIRBUS/ONERA airfoils in near stall conditions. Part I: Boundary layers," Aerospace Science and Technology, Vol. 7, No. 6, 2003, pp. 439-449.

${ }^{22}$ Schüle, C. Y. and Rossignol, K.-S., "Trailing-edge noise modeling and validation for separated flow conditions," $19^{t h}$ AIAA/CEAS Aeroacoustics Conference, Berlin, Germany, AIAA 2013-2008, 2013.

${ }^{23}$ Suryadi, A. and Herr, M., "Wall Pressure Spectra on a DU96-W-180 Profile From Low to Pre-Stall Angles of Attack," 21 ${ }^{\text {st }}$ AIAA/CEAS Aeroacoustics Conference, Dallas, Texas, AIAA 2015-2688, 2015.

${ }^{24}$ Herr, M., Trailing-Edge Noise - Reduction Concepts and Scaling Laws, Ph.D. thesis, Technische Universität Braunschweig, Braunschweig, Germany, 2013, DLR Forschungbericht 2013-32, ISSN 1434-8454. 